Bull. Chem. Soc. Ethiop. 2017, 31(1), 1-15.

ISSN 1011-3924

(c) 2017 Chemical Society of Ethiopia and The Authors

Printed in Ethiopia

DOI: http://dx.doi.org/10.4314/bcse.v31i1.1

\title{
THE QUEChERS ANALYTICAL METHOD COMBINED WITH LOW DENSITY SOLVENT BASED DISPERSIVE LIQUID-LIQUID MICROEXTRACTION FOR QUANTITATIVE EXTRACTION OF MULTICLASS PESTICIDE RESIDUES IN CEREALS
}

\author{
Tesfa Bedassa ${ }^{1,2}$, Abera Gure ${ }^{1,3}$ and Negussie Megersa ${ }^{1 *}$ \\ ${ }^{1}$ Department of Chemistry, Addis Ababa University, P. O. Box 1176, Addis Ababa, Ethiopia \\ ${ }^{2}$ Permanent Address: Department of Chemistry, Wollega University, P. O. Box 395, Nekemte, \\ Ethiopia \\ ${ }^{3}$ Permanent Address: Department of Chemistry, Jimma University, P. O. Box 378, Jimma, \\ Ethiopia
}

(Received May 13, 2016; revised March 28, 2017)

\begin{abstract}
In this study, a combination QuEChERS and low density solvent based DLLME was developed for extraction and preconcentration of five multiclass pesticide residues including atrazine, ametryn, terbutryn, carbaryl and chlorothalonil, from selected cereals: Teff, barley and maize samples. Liquid chromatography with variable wavelength detector (VWD) was used for quantitative determination of the pesticides. Various experimental parameters affecting the extraction and the preconcentration efficiencies were optimized. Calibration curves constructed using Teff samples were linear over wide ranges, 7.1-200 $\mu \mathrm{g} \mathrm{kg}^{-1}$ with $\mathrm{R}^{2} \geq 0.990$. LODs of the method were $\leq 8.9 \mu \mathrm{g} \mathrm{kg}^{-1}$, below the MRLs of EU for maize and barley. The RSDs were in the range of 2.3$9.5 \%$. The method has also been applied to different cereal samples and satisfactory average recoveries ranging $69.0-117 \%$ were obtained for the spiked cereal samples at two concentration levels. Therefore, the developed method can be successfully utilized as a simple alternative for the analysis of multiclass pesticide residues in cereals and other related samples.
\end{abstract}

KEY WORDS: QuEChERS method, Low density solvent based DLLME, Multiclass pesticide residues, Cereal samples, HPLC-VWD, Quantitative extraction

\section{INTRODUCTION}

The great demand for cereals, as well as their processed products, to feed the ever-growing population of the world, requires considerable efforts to improve their production yields, especially those that are significantly affected by pests. As a result, various classes of pesticides including insecticides, fungicides and herbicides, are regularly applied to cereals $[1,2]$. Use of pesticides, on the other hand, could result in leftover residues on the cereal yields. For food safety and environmental reasons, legislative directives and monitoring programs of different countries; for example, USA, China, India and the EU have set maximum residue limits (MRLs) of pesticide residues in various raw and processed foods, including cereals [2-4].

Analysis of multiclass pesticide residues in foods is a challenging task because of the different physicochemical properties of the pesticides, complexity of the matrices and low concentrations at which they are usually occurring [5,6]. Though great advances have been made on the development of highly efficient and sensitive analytical techniques for the final determination of these compounds, the use of efficient sample preparation methods is still greatly momentous in order to obtain accurate quantitative results, at or below MRLs of these compounds set by regulatory bodies.

Several sample preparation methods have been developed for extraction and/or prconcentration of multiclass pesticide residues in various cereals and other food samples. The methods include solvent extraction (SE) or liquid-liquid extraction (LLE) [7], solid-phase

*Corresponding author. E-mail: megersane@yahoo.com, negussie.megersa@aau.edu.et 
extraction (SPE) [8, 9], supported liquid membrane extraction (SLM) [10], matrix solid-phase dispersion (MSPD) [11, 12], solid-phase microextraction technique (SPME) [13, 14], supercritical fluid extraction (SFE) [15], microwave-assisted extraction (MAE) [16] and pressurized liquid extraction (PLE) [17, 18]. However, these sample preparation methods have several disadvantages; they are time-consuming, require high volume of expensive and/or toxic organic solvents and methods such as SFE and PLE demands expensive equipments. Besides, methods such as SE or LLE, SPE and SPME are not convenient to be used for processing solid samples, unless other time consuming sample pretreatment techniques are considered.

Recently, the Quick, Effective, Cheap, Effective, Rugged and Safe (QuEChERS) technique was developed, primarily for extraction of multiclass pesticide residues in non-fatty matrices such as sugarcane honey [19], honey [20, 21], fruits and vegetables [22, 23] and its application has subsequently been extended to the extraction of multiclass pesticide residues from high fat matrices such as raw cereals and their processed products [23-30]. The QuEChERS method eliminates several time consuming and complicated sample pretreatment steps commonly employed in traditional methods [2]. However, due to the low water content of cereal samples (below 25\%), water should be added to the samples with acetonitrile (the extraction solvent) in order to increase the moisture content to above $75 \%$, with the objective of weakening the possible interactions of the analytes with the sample matrix, and thus consequently, improving the efficiency of the extraction process [28]. On the other hand, although QuEChERS procedure nowadays, is a method of choice for analysis of multiclass pesticides residues in various solid and semi-solid samples, it does not involve preconcentration (enrichment) of the target analytes $[31,32]$. Quantitative determination of the final extract of the sample has commonly been performed utilizing highly sensitive analytical techniques such as gas (GC) and/or liquid chromatography (LC) coupled with mass spectrometry (MS) or tandem mass spectrometric (MS/MS) detectors [3, 23, 25, 30, 33, 34].

In the last few years, the use of combination of QuEChERS and dispersive liquid-liquid micro-extraction (DLLME) for analysis of multiclass pesticides resides in food samples have been reported by a number of workers [32, 35-39]. The introduction of DLLME to the QuEChERS procedure was found to increase the enrichment factor of the extraction processes. However, the extraction solvents, which were reported so far in DLLME procedures, are those having higher density than water such as halogenated hydrocarbons, but, these solvents are potentially toxic to human beings and the environment. To the best of our knowledge, the combination of QuEChERS with low density solvent DLLME (LDS-DLLME) based extraction and preconcentration methodology has not yet been reported for analysis of multiclass pesticides in cereal samples.

Therefore, in this study, QuEChERS-LDS-DLLME method has been proposed for quantitative extraction and preconcentration of five multiclass pesticides residues in three cereals including Teff (Eragrostis tef), maize and barley, utilizing high performance liquid chromatography with variable wavelength detector (HPLC-VWD). Teff is an ancient and a major cereal crop, indigenous to Ethiopia [39-41], from which a staple food, "Injera", is prepared. "Injera" is known to provide approximately two third of the diet for the Ethiopian population [41]. Similarly, maize and barley are also the cereals most commonly used for preparing various types of foods all over the country. The pesticides considered in this study comprises of atrazine (Atraz), ametryn (Amet), and terbutryn (Terb) among herbicides; carbaryl (Carb) from insecticides; and chlorothalonil (Chlor) which is a fungicide. In order to ensure reliability of the proposed technique, various parameters affecting the extraction efficiency as well as the chromatographic separation performances during the analysis of the target analytes have been investigated so as to establish the optimum conditions for extensive future applications. 
Chemicals and reagents

\section{EXPERIMENTAL}

All chemicals and reagents used in this study were of analytical grade and the solvents, used both as mobile phase and extraction solvents, were of HPLC grade. Analytical pesticide standards of Carb and Chlor were purchased from Sigma Aldrich (St. Louis, MO, USA). Atr, Amet and Terb were obtained from Dr. Ehrenstorfer (Augusburg, Germany). The chemical structures, common names, abbreviations and the $\mathrm{pK}_{\mathrm{a}}$ of the target pesticides are given in Figure 1. Anhydrous magnesium sulphate $\left(\mathrm{MgSO}_{4}\right)$ was purchased from Fisher Scientific Company L. C. C. (Pittsburgh, USA). Sodium chloride $(\mathrm{NaCl})$, glacial acetic acid $\left(\mathrm{CH}_{3} \mathrm{COOH}\right)$ and anhydrous sodium acetate $\left(\mathrm{CH}_{3} \mathrm{COONa}\right)$ were obtained from $\mathrm{BDH}$ Laboratory supplies (Poole, England). Acetonitrile was purchased from Ashland Chemical (S. Giuliano MI, Italy) and ultrapure water obtained after purification with double distiller A8000 Aquatron water still (Bibby Scientific Ltd, Staffordshire, UK) was used throughout the study.

\section{Preparation of pesticides standard solutions}

Individual stock standard solutions containing $1000 \mathrm{mg} \mathrm{L}^{-1}$ were prepared separately by dissolving accurately weighed amount of each standard in acetonitrile. Intermediate standard solution containing $20 \mathrm{mg} \mathrm{L}^{-1}$ of each standard was also prepared from the stock solutions by diluting with acetonitrile. Working standard solutions were prepared from the mixed intermediate standard solution by diluting with deionized water and then used for optimization of the parameters affecting the QuEChERS-DLLME procedure as well as method validation. All solutions were stored under refrigeration below $4{ }^{\circ} \mathrm{C}$ when not in use.<smiles>CNC(=O)Oc1cccc2ccccc12</smiles>

Carbaryl (Carb)

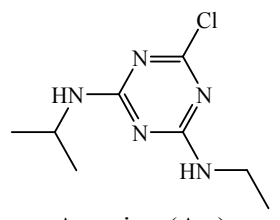

Atrazine (Atr)

$\mathrm{pK}_{\mathrm{a}} 10.4 ; \log \mathrm{P} 2.36$

$\mathrm{pK}_{\mathrm{a}} 1.7 ; \log \mathrm{P} 2.7$

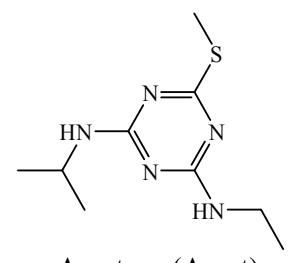

Ametryn (Amet)<smiles>CCNc1nc(NC(C)(C)C)nc(SC)n1</smiles>

Terbutryn (Terb)

$\mathrm{pK}_{\mathrm{a}} 4.3 ; \log \mathrm{P} 3.7$

$$
\mathrm{pK}_{\mathrm{a}} \text { 10.07; } \log \mathrm{P} 2.63
$$<smiles>N#Cc1c(Cl)c(Cl)c(Cl)c(C#N)c1Cl</smiles>

Chlorothalonil (Chlor)

$\log$ P 2.94

Figure 1. The chemical structures, common names, abbreviations and $\log \mathrm{P}$ (octanol-water partition coefficients) at $\mathrm{pH} 7$ and $20^{\circ} \mathrm{C}$ of the target pesticides.

\section{Instruments and equipment}

Chromatographic analyses were performed using Agilent Technologies 1200 infinity series HPLC, equipped with quaternary pump, vacuum degasser, autosampler and UV-Vis variable 
wavelength detector (VWD); all purchased from Agilent Technologies (Waldbronn, Germany). Chromatographic separation was carried out using Eclipse plus $C_{18}$ column (100 x $4.6 \mathrm{~mm} \mathrm{I}$. D., $3.5 \mu \mathrm{m}$ particle sizes) obtained from Agilent Technologies. Data acquisition and processing were accomplished with LC Chemstation software (Agilent Technologies).

The d-SPE tubes, Supel QuE PSA (EN) Tubes, containing $150 \mathrm{mg}$ supelclean PSA, $150 \mathrm{mg}$ Discovery DSC-18 and $900 \mathrm{mg} \mathrm{MgSO}_{4}$, used for sample clean-up in QuEChERS extraction procedure were purchased from Sigma-Aldrich (St. Louis, MO, USA). The ultrasonic cleaner Decon ${ }^{\circledR}$, from Decon Laboratories Limited (Hove, East Sussex); a centrifuge, Centurion Scientific Limited (Ford, Arundel, West Sussex); a centrifuge, model 800, Jiangsu Zhenji Instruments Co., Ltd. (Jiangsu, China); and $50 \mathrm{~mL}$ centrifuge tube, (Corning Inc., NY, USA) were used during sample preparation.

\section{Chromatographic conditions}

The chromatographic separation of the target analytes was performed based on a previous paper [32] with minor modifications. An isocratic elution with a binary mobile phase comprising $45 \%$ water (solvent A) and 55\% acetonitrile (solvent B) was used throughout the analysis. Prior to the subsequent sample/extract injection, the HPLC column was washed by adjusting the mobile phase composition to 5\% water (solvent A) and 95\% acetonitrile (solvent B) for 15 min and then was conditioned with the mobile phase for additional $5 \mathrm{~min}$. Analysis was performed with the mobile phase flow rate of $0.3 \mathrm{~mL} \mathrm{~min}^{-1}$, column temperature set at $30^{\circ} \mathrm{C}$, injection volume 10 $\mu \mathrm{L}$ and monitoring wavelength of $224 \mathrm{~nm}$.

\section{Cereal samples}

Three different types of cereal samples; namely, Teff, maize and barley, which are commonly consumed in Ethiopia, were collected from two different localities: from Addis Ababa (Central Ethiopia and capital of the country), in ground form, thus obtained as flour; and from local market in Nekemte (Western Ethiopia; Oromia National Regional State). The cereals collected from Nekemte were also milled in Addis Ababa to obtain similar degree of fineness.

\section{QuEChERS-LDS-DLLME extraction procedure}

The extraction procedure of Teff consisted of two steps; QuEChERS and DLLME. (i) The QuEChERS procedure was performed following the literature reports [28, 29] with some modifications. Accordingly, $21 \mathrm{~g}$ Teff flour was weighed in a beaker to which $49 \mathrm{~mL}$ water was added, and then the content was thoroughly mixed with a spatula to make a dough, containing Teff to water mass ratio of approximately $3: 7$. Then, $10 \mathrm{~g}$ of the dough sample was transferred to a 50-mL centrifuge tube and subsequently spiked with appropriate concentrations of the target analyte standard mixtures. The content was then shaken for few seconds and kept to stand for about $30 \mathrm{~min}$ to establish equilibrium. After addition of $5 \mathrm{~mL}$ acetonitrile, the content was shaken for few more seconds. This was followed by addition of $4 \mathrm{~g} \mathrm{MgSO}_{4}$ and $1 \mathrm{~g} \mathrm{NaCl}$ to the sample content and further shaken vigorously for $1 \mathrm{~min}$. Then, after centrifuging the resulting content at $3000 \mathrm{rpm}$ for $10 \mathrm{~min}, 3 \mathrm{~mL}$ of the upper layer of the acetonitrile extract was transferred to the d-SPE tube. The d-SPE tube was then sealed, shaken for about 1 min and was centrifuged at $4000 \mathrm{rpm}$ for $3 \mathrm{~min}$. Finally, $1 \mathrm{~mL}$ of the resulting acetonitrile extract was transferred into a $4 \mathrm{~mL}$ vial and mixed with $125 \mu \mathrm{L}$ dihexyl ether for subsequent DLLME extraction procedure.

(ii) For the DLLME procedure, $5 \mathrm{~mL}$ distilled water containing $10 \% \mathrm{NaCl}(w / v)$ was first taken into $15 \mathrm{~mL}$ centrifuge tube. Thereafter, a mixture of $1 \mathrm{~mL}$ acetonitrile extract (used as a disperser), obtained from the earlier QuEChERS extraction procedure, and $125 \mu \mathrm{L}$ dihexyl ether 
(extraction solvent) were rapidly injected into the content using $5 \mathrm{~mL}$ syringe with a sharp needle, to induce a cloudy solution formation due to the dispersion of fine droplets of the extraction solvent in the water sample. The content was then centrifuged at $4000 \mathrm{rpm}$ for $5 \mathrm{~min}$ and then the organic phase was separated as clear solution (upper layer) from the surface of the aqueous phase (bottom layer). The lower aqueous phase was then removed using a $5 \mathrm{~mL}$ syringe, leaving volume equivalent to the organic phase at the bottom of the tube, which was convenient for collection of the organic phase. Finally, $50 \mu \mathrm{L}$ of the organic phase was conveniently collected, using a micropipette and diluted with $100 \mu \mathrm{L}$ acetonitrile in the insert vial, which was housed in a $1.5 \mathrm{~mL}$ autosampler vial, to be transferred to the HPLC autosampler for subsequent injection of $10 \mu \mathrm{L}$.

Similarly, for maize and barley samples the same procedures described above for Teff sample were followed, with minor modification on the QuEChERS procedure, particularly in the mass ratios of the flours to the volume of water used for dough preparations. Therefore, maize and barley doughs were formed by mixing $3 \mathrm{~g}$ of their flours in $8 \mathrm{~mL}$ of water.

\section{RESULTS AND DISCUSSION}

\section{Optimization of the extraction conditions}

In this study, the combination of QuEChERS and LDS-DLLME was proposed for extraction and preconcentration of target pesticides from three cereal samples including Teff, maize and barley. Quantitative determination of the analytes in the final sample extracts was achieved by HPLC-VWD following the conditions reported earlier for determination of target analytes [32] and the QuEChERS procedure [28, 29] with some minor modifications, especially on the ratio of the masses of the samples to the volume of water. In principle, addition of water to the cereal samples is required in order to make the sample matrices more accessible to the extraction solvent [33]. Accordingly, the ratio of the mass of Teff flour to the volume of water was set after investigating the quality of dough obtained by mixing in various proportions. Eventually, the optimized ratio selected for the target samples were $3 \mathrm{~g}$ Teff flour to $7 \mathrm{~mL}$ water and $3 \mathrm{~g}$ maize and barley flours to $8 \mathrm{~mL}$ water. To preconcentrate the extracted analytes by the QuEChERS procedure, LDS-DLLME was employed because of its simplicity, low cost, speed, novelty and benignity to the environment [42].

In order to establish the optimum LDS-DLLME conditions, different parameters affecting the extraction efficiency of the technique were studied. These variables include the type and volume of extraction and the disperser solvents, $\mathrm{pH}$ of the aqueous samples and the ionic strength [42]. In these experiments, the peaks are as in milliabsorbance units (mAU) of the analytes were used to evaluate the influences of the selected factors on performance of the method. All the experiments were performed in triplicate with appropriate concentration of the standard solution containing the five target multiclass pesticides, and averages of the replicate analyses were taken for optimization of the different experimental parameters.

\section{Selection of the extraction solvent}

In the LDS-DLLME enrichment procedure, selection of appropriate extraction solvent is often crucial for obtaining satisfactory preconcentration of the target analytes. The solvent chosen should have lower density than water, appreciable capability of preconcentrating the target analytes, low solubility in water and good chromatographic behavior under selected HPLC conditions [43]. To this end, six organic solvents, with their densities given in parentheses, including dihexyl ether $\left(0.785 \mathrm{~g} \mathrm{~mL}^{-1}\right)$ n-octanol $\left(0.827 \mathrm{~g} \mathrm{~mL}^{-1}\right)$, toluene $\left(0.865 \mathrm{~g} \mathrm{~mL}^{-1}\right)$, undecane $\left(0.740 \mathrm{~g} \mathrm{~mL}^{-1}\right)$, cyclohexane $\left(0.811 \mathrm{~g} \mathrm{~mL}^{-1}\right)$ and $\mathrm{n}$-heptane $\left(0.680 \mathrm{~g} \mathrm{~mL}^{-1}\right)$ were 
investigated as potential extraction solvents. The extraction was carried out by injecting the mixtures of $100 \mu \mathrm{L}$ each of the solvents with $1000 \mu \mathrm{L}$ acetonitrile as the disperser. With the exception of cyclohexane, all the selected extraction solvents exhibited satisfactory phase separation. However, when the sample extract obtained utilizing toluene was injected, a broad peak which might be attributed to toluene was observed in the chromatogram at the retention time of Terb. Furthermore, with 1-octanol the peaks of the last three analytes, i.e. Amet, Terb, and Chlor, were also lost, probably not partitioned between the phases, and may be entirely retained in the solvent. The results obtained using dihexyl ether; heptane and undecane are shown in Figure 2. Dihexyl ether exhibited the highest extraction performance for all the target analytes and was thus selected as the extraction solvent in the subsequent experiments.

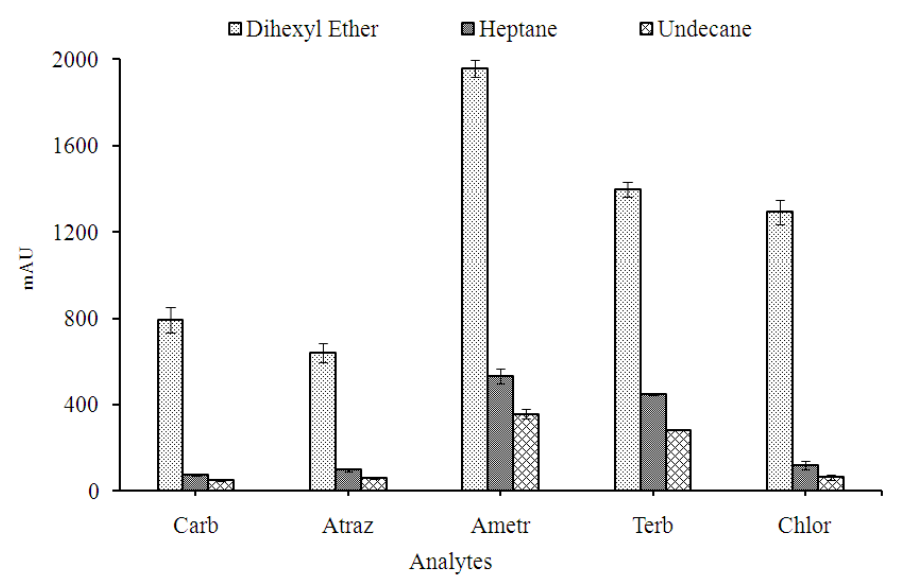

Figure 2. Effect of the type of extraction solvent. Experimental conditions: volume of the extraction solvent, $100 \mu \mathrm{L}$; volume of the disperser solvent (acetonitrile), $1000 \mu \mathrm{L} ; 5 \%$ $(w / v) \mathrm{NaCl}$, centrifugation speed and time, $4000 \mathrm{rpm}$ and $5 \mathrm{~min}$, respectively.

Selection of the volume of the disperser solvent

In order to obtain the optimum volume of the dispenser solvent, various volumes (in the range of $800-1400 \mu \mathrm{L}$ ) of acetonitrile extract, obtained from QuEChERS procedure containing a fixed volume of dihexyl ether (i.e. $100 \mu \mathrm{L}$ ) were investigated. It was observed that the peak areas of all the target analytes were increased with increasing the volume of methanol up to $1000 \mu \mathrm{L}$ and then decreased for three of the analytes including Carb, Atraz and Chlor (Figure 3). At low volumes of the disperser solvent volumes, the cloudy state was not properly formed and thus resulted in low extraction performances. Similarly, use of higher volumes of acetonitrile was also found to decrease the extraction efficiency. This could be associated to the possible enhanced solubility of the target analytes in aqueous phase due to an increase in partitioning of the dispersive solvent in the aqueous sample [44, 45]. As a result, $1000 \mu \mathrm{L}$ methanol was chosen for the subsequent experiments.

Selection of volume of the extraction solvent

Volume of the extraction solvent is another crucial parameter influencing the extraction and/or preconcentration efficiency of the proposed LDS-DLLME procedure. With increasing the volume of extraction solvent, volume of the upper organic phase obtained after extraction will 
also increase, resulting in reduction of the enrichment factors of the target analytes [35]. Therefore, the optimal volume of extraction solvent should offer high enrichment factors as well as sufficient volume of the organic phase for subsequent analysis.

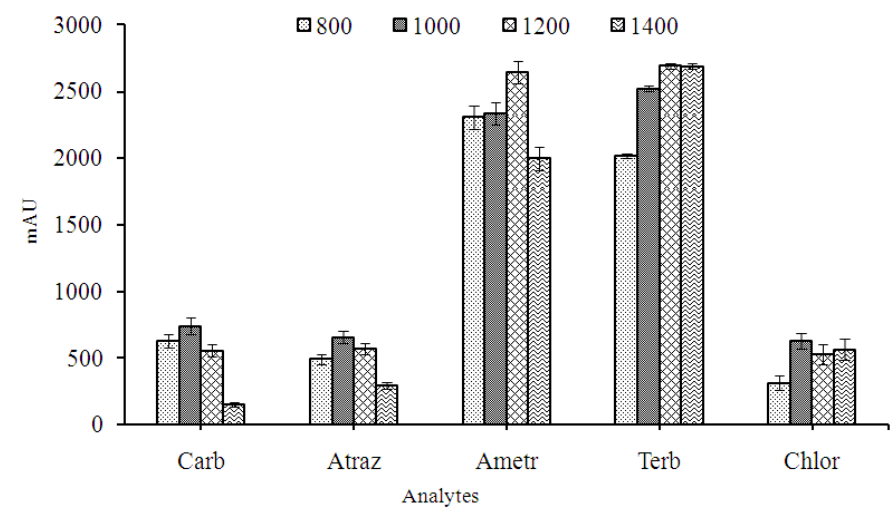

Figure 3. Effect of the disperser solvent volume. All other experimental conditions remain the same as those in Figure 2.

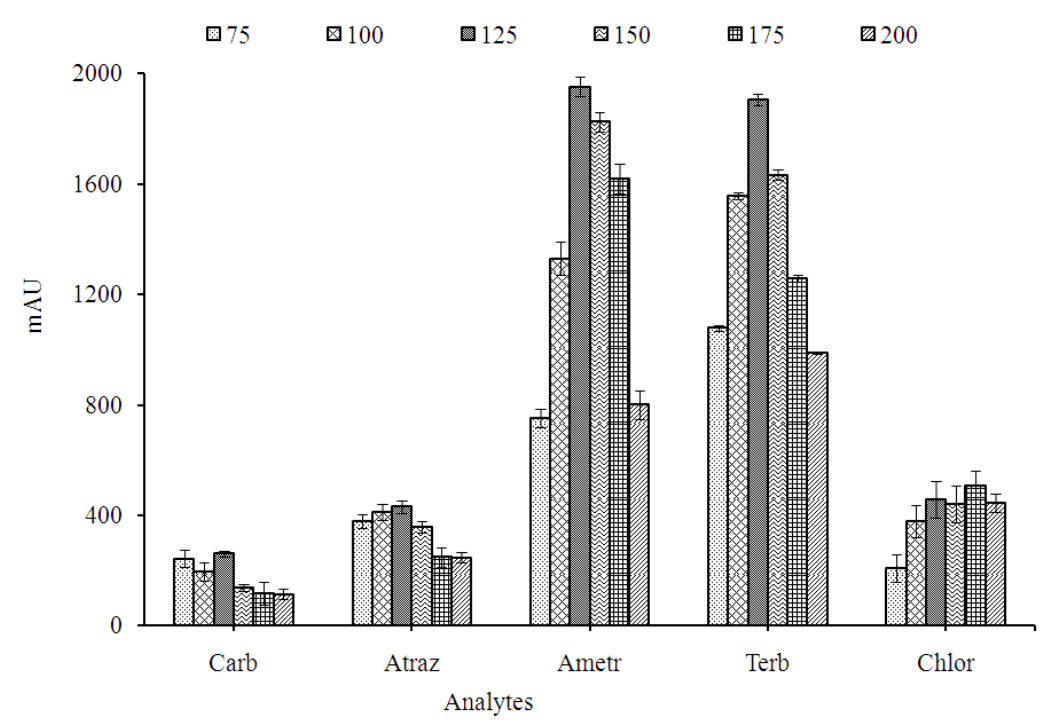

Figure 4. Effect of extraction solvent volume. Experimental conditions: disperser solvent volume, $1000 \mu \mathrm{L}$; and all other experimental conditions remain the same as those in Figure 2.

In this study, the effect of extraction solvent volume on extraction efficiency of the LDSDLLME technique was evaluated by varying the volume of dihexyl ether over the range of 100$200 \mu \mathrm{L}$, while the other experimental parameters were kept constant. As can be seen from Figure 4, the peak areas of all the target analytes increased with the volume of extraction solvent up to $125 \mu \mathrm{L}$ and then lowered at higher volumes. With lower volumes, the extraction solvent 
may not properly be dispersed and thus as a consequence, the transfer of the analytes from aqueous sample to the organic phase decreases. On the other hand, the decrease in the peak areas of the target analytes at higher volumes of the extraction solvent could be attributed to the reduction of the enrichment factors (or dilution effect), resulting from the increase in the volume of organic phase collected after extraction [35, 46, 47]. Thus, $125 \mu \mathrm{L}$ of diexhyl ether was chosen as the optimum volume in all the subsequent experiments.

\section{Effect of salt addition}

In general, addition of a small amount of salt into the sample solution produces a salting out effect, decreasing the solubility of the analytes in the aqueous phase and thus enhances the transfer of analytes into the organic phase [45]. The effect of addition of the salt, on the extraction performance of LDS-DLLME was investigated by varying the amounts of $\mathrm{NaCl}(0-$ $15 \%, w / v)$, keeping all the other experimental conditions constant. It was observed that the peak area of all target analytes increased with the quantity of the salt added up to $10 \%$ and was then remained nearly constant on addition of larger quantities of the salt. Thus, $10 \%(w / v) \mathrm{NaCl}$ was selected to be optimum concentration in all subsequent experiments.

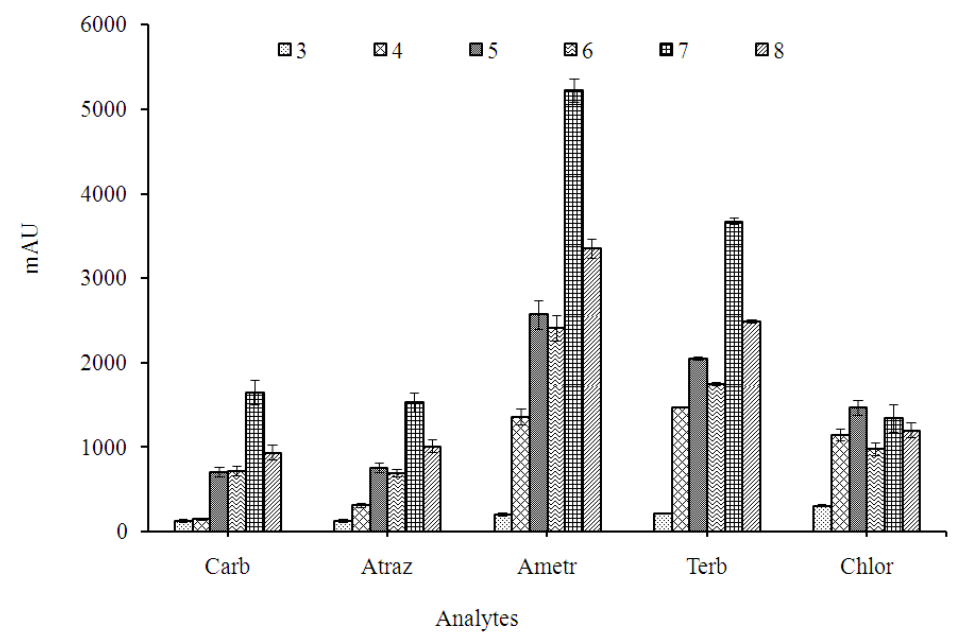

Figure 5. Effect of sample pH. Experimental conditions: extraction solvent volume, $125 \mu \mathrm{L}$; $\mathrm{NaCl}$ concentration, $10 \%$; disperser solvent volume, $1000 \mu \mathrm{L}$ and all other conditions remained the same as those in Figure 2.

\section{Effect of the sample $p H$}

With the exception of Chlor, all the target analytes considered in this study are ionizable pesticides. Thus, to extract such substances into the organic phase, the $\mathrm{pH}$ of the sample solution should be adjusted to minimize their ionizability and consequently reduce their solubility in the aqueous phase [48, 49]. The effect of sample $\mathrm{pH}$ on the extraction efficiencies of the target analytes were evaluated by varying its values from 3 to 8 using phosphate buffer. It was observed that the peaks of all target analytes were increased with increasing $\mathrm{pH}$ up to 7 and was then decreased at higher $\mathrm{pH}$ values (Figure 5). Thus, $\mathrm{pH} 7$ was selected as the optimum value for subsequent experiments. 


\section{Analytical performance characteristics}

The analytical performance of the proposed QuEChERS-LDS-DLLME method was investigated using matrix matched calibration curves, established in Teff samples free from the target pesticides as representative matrix. The calibration curves were constructed by spiking the Teff samples with a mixture of the target analytes at five concentration levels, corresponding to 20 , $50,100,150$, and $200 \mu \mathrm{g} \mathrm{kg}^{-1}$. Each concentration level was extracted in duplicate, and each extract was also injected in duplicate. The calibration curves were then obtained by plotting the chromatographic peak areas as the instrumental response versus the target analytes concentrations. It was observed that the proposed method exhibited good linearity over the studied concentrations ranges, with coefficients of determination of 0.990 or better. The limits of detection (LOD) and limit of quantification (LOQ), which were determined as the concentrations that give 3 and 10 times the signal to noise ratio $(\mathrm{S} / \mathrm{N})$ were in the range of 1.8 $8.9 \mu \mathrm{g} \mathrm{kg}^{-1}$ and 7.1-29.6 $\mu \mathrm{g} \mathrm{kg}^{-1}$, respectively, and found to be below the European maximum residue limits (MRLs) set for these analytes in cereal samples such as maize and barley [4]. The performance characteristics of the proposed method in the Teff sample are shown in Table 1.

Table 1.Analytical performance characteristics of the proposed QuEChERS-LDS-DLLME method.

\begin{tabular}{|c|c|c|c|c|c|}
\hline Analytes & Linear range $\left(\mu \mathrm{g} \mathrm{L}^{-1}\right)$ & $\mathrm{R}^{2}$ & $\mathrm{LOD}(\mu \mathrm{g} \mathrm{kg}$ & $\mathrm{LOQ}(\mu \mathrm{g} \mathrm{kg}$ & EU MRL $\left.^{-1} \mu \mathrm{g} \mathrm{kg}^{-1}\right)$ \\
\hline Carb & $30-200$ & 0.998 & 8.9 & 29.6 & 500 \\
\hline Atraz & $23-200$ & 0.999 & 6.9 & 22.9 & 100 \\
\hline Amet & $11-200$ & 0.996 & 3.2 & 10.7 & - \\
\hline Terb & $8-200$ & 0.995 & 2.1 & 7.1 & - \\
\hline Chlor & $6-200$ & 0.997 & 1.8 & 5.9 & 10 \\
\hline
\end{tabular}

\section{Precision study}

The precision of the proposed method was studied in terms of intra-day precision (repeatability) and inter-day precision (reproducibility) by applying the optimized conditions. Intra-day precision was evaluated by spiking the Teff samples at two concentration levels: Level 1, $50 \mu \mathrm{g}$ $\mathrm{kg}^{-1}$ and Level 2, $150 \mu \mathrm{g} \mathrm{kg}^{-1}$. Each concentration level was prepared in duplicate (experimental replicate) and was then injected in triplicate (instrumental replicate) on the same day, under the same experimental conditions. Likewise, inter-day precision was also assessed by extracting one Teff sample at each of the two concentration levels utilized for intra-day precision studies, for three consecutive days and each concentration level was then injected in triplicate. Table 2, shows the obtained results of both intra- and inter-day precisions, presented as the relative standard deviations (RSD) of the peak areas. The obtained precision results were less than $10 \%$, which is within acceptable precision range established by the European Commission for pesticide residue analysis in food and feed samples [50].

Table 2. Intra- and inter-day precisions of the proposed method for the spiked Teff samples.

\begin{tabular}{|c|c|c|c|c|}
\hline \multirow{2}{*}{ Analytes } & \multicolumn{2}{|c|}{ Intra-day RSD $(\mathrm{n}=6)$} & \multicolumn{2}{c|}{ Inter-day RSD $(\mathrm{n}=9)$} \\
\cline { 2 - 5 } & Level 1 & Level 2 & Level 1 & Level 2 \\
\hline Carb & 6.0 & 4.8 & 9.5 & 6.9 \\
\hline Atraz & 6.3 & 2.6 & 5.0 & 5.3 \\
\hline Amet & 3.3 & 7.7 & 7.3 & 8.4 \\
\hline Terb & 4.5 & 2.3 & 8.8 & 7.9 \\
\hline Chlor & 3.0 & 4.2 & 3.6 & 7.9 \\
\hline
\end{tabular}

Level 1: $50 \mu \mathrm{g} \mathrm{kg}^{-1}$; Level 2: $150 \mu \mathrm{g} \mathrm{kg}^{-1}$. 
Applications and recovery studies

The practical applicability of the proposed method was evaluated by performing recovery studies in three different types of cereals comprising of Teff, maize and barley samples. None of the target analytes was detected in any of these samples. The recovery studies were performed at two concentration levels, similar to those earlier used for precision study. Each concentration level was extracted in duplicate, and each was then injected in triplicate. Recoveries were calculated by comparing the concentration of the extracted analytes with the initial concentration of the target analytes, spiked to the cereal samples [37]. Recoveries and the corresponding RSD $(\mathrm{n}=6)$ of each target analyte in Teff, maize, and barley samples are shown in Table 3. The observed recoveries were in the range of $69-117 \%$ with RSD ranging $2.3-9.8 \%$, in all the target analytes. These results were in good agreement with the acceptable recovery range established by the European Commission for pesticide residue analysis in food and feed samples [50].

Table 3. Percentage recoveries $(\% \mathrm{R}, \mathrm{n}=6)$ of the method for Teff, maize, and barley samples.

\begin{tabular}{|c|c|c|c|c|c|c|}
\hline \multirow{2}{*}{ Analyte } & \multicolumn{2}{|c|}{ "Teff”, \%R (RSD) } & \multicolumn{2}{c|}{ Maize, \%R (RSD) } & \multicolumn{2}{c|}{ Barley, \%R (RSD) } \\
\cline { 2 - 7 } & Level 1 & Level 2 & Level 1 & Level 2 & Level 1 & Level 2 \\
\hline Carb & $95(6.0)$ & $101(4.8)$ & $70(7.6)$ & $76(9.8)$ & $78(6.5)$ & $111(8.9)$ \\
\hline Atraz & $95(6.3)$ & $99(2.6)$ & $71(8.7)$ & $73(7.9)$ & $69(8.8)$ & $84(8.1)$ \\
\hline Amet & $101(3.3)$ & $93(7.7)$ & $107(5.5)$ & $88(5.5)$ & $102(7.7)$ & $101(7.7)$ \\
\hline Terb & $107(4.5)$ & $95(2.3)$ & $107(4.3)$ & $84(8.0)$ & $104(6.9)$ & $96(5.0)$ \\
\hline Chlor & $115(3.0)$ & $97(4.2)$ & $69(6.7)$ & $70(4.9)$ & $115(6.9)$ & $117(4.8)$ \\
\hline
\end{tabular}

Level 1: $50 \mu \mathrm{kg}^{-1}$; level 2: $150 \mu \mathrm{gg}^{-1}$.

Typical representative chromatograms of the blank maize and of the sample spiked with 500 $\mu \mathrm{g} \mathrm{kg} \mathrm{kg}^{-1}$ for carb, atraz, amet, terb, and chlor, analyzed by the proposed QuEChERS-LDSDLLME combined with HPLC-VWD method under the optimum conditions are shown in Figure 6.

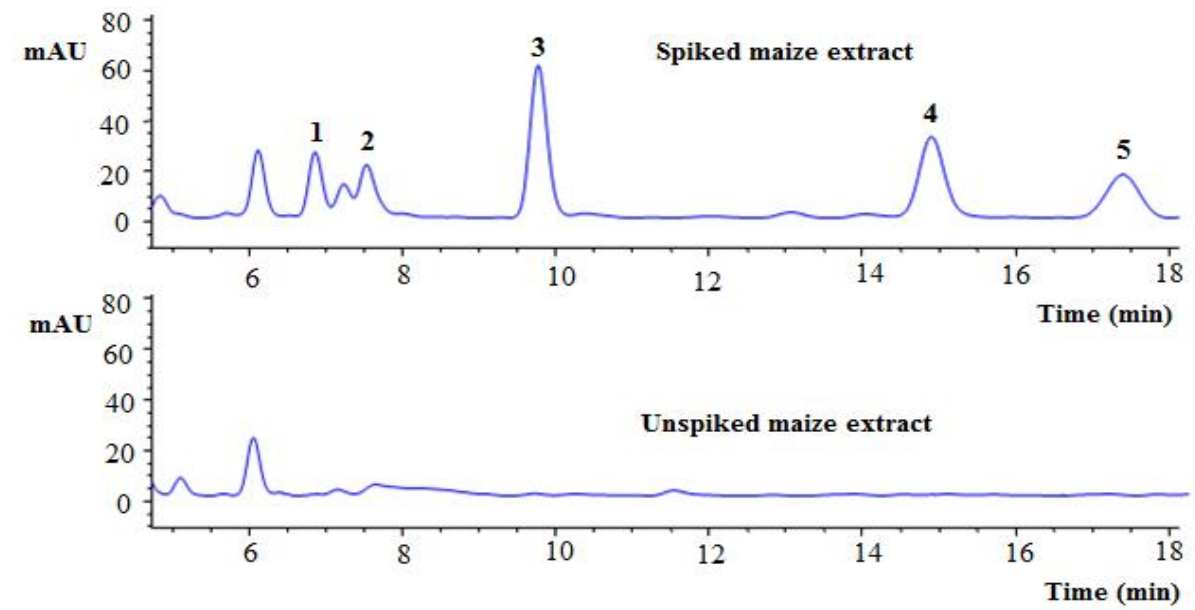

Figure 6. Typical chromatograms of the blank and spiked maize samples with $500 \mu \mathrm{g} \mathrm{kg}^{-1}$ of all the target analytes. (1) Carb, (2) Atraz, (3) Ametr, (4) Terb and (5) Chlor. 
Comparison of the proposed method with other reported methods

The extraction efficiency of the proposed QuEChERS-LDS-DLLME procedure has been compared with other similar techniques recently reported utilizing QuEChERS [3, 23, 25, 30, 34] and QuEChERS-DLLME [47] in terms of the parameters such as linearity ranges, LOD, and $\%$ R. Details of the comparison are shown in Table 4 . The proposed method was found to exhibit similar or better linearity ranges, better or comparable LOQs and recoveries values than the other reported ones. The method also utilizes less toxic organic solvent and relatively inexpensive analytical technique, which could be accessible in common research laboratories. Based on the experimental findings and the deduction from the comparison, the proposed method is simpler, cheaper, more rapid and environmentally benign alternative for the trace level extraction and quantitative determination of multiclass pesticide residues in cereals and other related matrices.

Table 4. Comparison of the proposed method with other reported methods for the extraction and determination of multiclass pesticide residues in maize sample.

\begin{tabular}{|c|c|c|c|c|c|c|c|}
\hline Analyte & Sample & $\begin{array}{c}\text { Extraction } \\
\text { method }\end{array}$ & $\begin{array}{c}\text { Analytic } \\
\text { method }\end{array}$ & $\begin{array}{c}\text { Linear range } \\
\left(\mu \mathrm{g} \mathrm{kg}^{-1}\right)\end{array}$ & $\begin{array}{c}\text { LOD } \\
\left(\mu \mathrm{g} \mathrm{kg}^{-1}\right)\end{array}$ & $\begin{array}{c}\text { Recovery } \\
(\% \mathrm{R})\end{array}$ & Reference \\
\hline $\begin{array}{c}122 \text { multiclass } \\
\text { pesticides }\end{array}$ & $\begin{array}{c}\text { Cereal grain and } \\
\text { certain feeding stuffs }\end{array}$ & QuEChERS & GC-MS/MS & $7.5-300$ & $2-10$ & $73-129$ & {$[3]$} \\
\hline 219 pesticides & $\begin{array}{c}\text { Cereals (corn, wheat } \\
\text { flour and rice })\end{array}$ & QuEChERS & GC-MS/MS & $2-200$ & - & $70-120$ & {$[23]$} \\
\hline 136 pesticides & Maize & QuEChERS & $\begin{array}{c}\text { UPLC- } \\
\text { MS/MS }\end{array}$ & $10-200$ & $<10-50$ & $70-120$ & {$[25]$} \\
\hline 16 herbicides & Milled polished rice & QuEChERS & LC-QqQ/MS & $5-500$ & $0.1-10$ & $70-120$ & {$[30]$} \\
\hline 124 pesticides & Rice & QuEChERS & GC-MS/MS & $10-200$ & $0.7-7.1$ & $70-122.7$ & {$[34]$} \\
\hline $\begin{array}{c}7 \\
\text { neonicotinoid } \\
\text { insecticides }\end{array}$ & $\begin{array}{c}\text { Cereal grains (brown } \\
\text { rice, maize, millet and } \\
\text { oat) }\end{array}$ & $\begin{array}{c}\text { QuEChERS- } \\
\text { DLLME }\end{array}$ & HPLC-DAD & $20-4,500$ & $2-5$ & $76-123$ & {$[47]$} \\
\hline $\begin{array}{c}5 \text { multiclass } \\
\text { pesticides }\end{array}$ & $\begin{array}{c}\text { Cereals (Teff, maize } \\
\text { and barley) }\end{array}$ & $\begin{array}{c}\text { QuEChERS- } \\
\text { LDS-DLLME }\end{array}$ & HPLC-VWD & $6-200$ & $1.8-8.9$ & $69-117$ & This work \\
\hline
\end{tabular}

\section{CONCLUSIONS}

In this study, an easy and environmentally safe sample extraction and/or preconcentration method has been proposed, by combining QuEChERS and LDS-DLLME procedures, for analysis of five multiclass pesticide residues including three herbicides (Atraz, Amet, and Terb), an insecticide (Carb) and one fungicide (Chlor), using HPLC-VWD from cereal samples; namely, Teff, barley and maize. The method has owed the advantages of high extraction efficiency, sensitivity, selectivity, and ease of operation with consumption of less toxic and low volume organic solvent. The use of LDS-DLLME after QuEChERS procedure considerably improved the preconcentration (enrichment) of the analytes compared to the methods that make use of the QuEChERS extraction method alone. The LODs and LOQs obtained were similar or better than the other reported findings on the analysis of multiclass pesticides in cereals and related matrices and also below the MRLs set by EU for pesticides residues in cereals. The proposed method has also provided wide linearity ranges, accepted precisions and satisfactory recoveries for all the target analytes in the studied cereal matrices. Therefore, the developed method could successfully be utilized as an attractive alternative for trace level extraction and determination of multiclass pesticide residues in cereals and other related matrices for quality control. 


\section{ACKNOWLEDGEMENTS}

Authors are grateful to the Department of Chemistry of the Addis Ababa University (AAU) for providing the laboratory facilities. Financial support for purchase of the analytical pesticide standards, HPLC grade solvents and other necessary expenses both for practical works as well as field studies were obtained from International Science Program (ISP) through "Trace Level Pollutants Analysis" project; ETH:04.

\section{REFERENCES}

1. Fenik, J.; Tankiewicz, M.; Biziuk, M. Properties and determination of pesticides in fruits and vegetables. Trends Anal. Chem. 2011, 30, 814-826.

2. Gonzalez-Curbelo, M.A.; Herrera-Herrera, A.V.; Ravelo-Perez, L.M.; Hernandez-Borges, J. Sample-preparation methods for pesticide-residue analysis in cereals and derivatives. Trends Anal. Chem. 2012, 38, 32-51.

3. Walorczyk, S. Development of a multi-residue screening method for the determination of pesticides in cereals and dry animal feed using gas chromatography-triple quadrupole tandem mass spectrometry. J. Chromatogr. A 2007, 1165, 200-212.

4. Regulation (EC) No. 149/2008 amending Regulation (EC) No. 396/2005 of the European Parliament and of the Council by establishing Annexes II, III and IV setting maximum residue levels for products covered by Annex I thereto. Official Journal of the European Communities, No. L 58, 1 March 2008, pp. 1-398.

5. Tadeo, J.L.; Sanchez-Brunete, C.; Perez, R.A.; Fernandez, M.D. Analysis of herbicide residues in cereals, fruits and vegetables. J. Chromatogr. A 2000, 882, 175-191.

6. Lambropoulou, D.A.; Albanis, T.A. Methods of sample preparation for determination of pesticide residues in food matrices by chromatography-mass spectrometry-based techniques: A review. Anal. Bioanal. Chem. 2007, 389, 1663-1683.

7. Lee, S.J.; Park, H.J.; Kim, W.; Jin, J.S.; Abd El-Aty, A.M.; Shim, J.H.; Shin, S.C. Multiresidue analysis of 47 pesticides in cooked wheat flour and polished rice by liquid chromatography with tandem mass spectrometry. Biomed. Chromatogr. 2009, 23, 434-442.

8. Chen, S.; Shi, L.; Shan, Z.; Hu, Q. Determination of organochlorine pesticide residues in rice and human and fish fat by simplified two dimensional gas chromatography. Food Chem. 2007, 104, 1315-1319.

9. Boccacci-Mariani, M.; Aiuto, V.D.; Giannetti, V. Multiresidue method for the determination of organophosphorus pesticides in cereal matrices. J. AOAC Int. 2010, 93, 999-1006.

10. Megersa, N.; Kassahun, S. A new selective liquid membrane extraction method for the determination of basic herbicides in agro-processed fruit juices and Ethiopian honey wine (Tej) samples. Food Addit. Contam. Part A 2012, 29,789-798.

11. Abhilash, P.C.; Singh, V.; Singh, N. Simplified determination of combined residues of lindane and other $\mathrm{HCH}$ isomers in vegetables, fruits, wheat, pulses and medicinal plants by matrix solid-phase dispersion (MSPD) followed by GC-ECD. Food Chem. 2009, 113, 267271.

12. Tsochatzis, E.D.; Menkissoglu-Spiroudi, U.; Karpouzas, D.G.; Tzimou-Tsitouridou, R.A multi-residue method for pesticide residue analysis in rice grains using matrix solid-phase dispersion extraction and high-performance liquid chromatography-diode array detection. Anal. Bioanal. Chem. 2010, 397, 2181-2190.

13. Djozan, D.; Mahkam, M.; Ebrahimi, B. Preparation and binding study of solid-phase microextraction fiber on the basis of ametryn-imprinted polymer application to the selective extraction of persistent triazine herbicides in tap water, rice, maize and onion. $J$. Chromatogr. A 2009, 1216, 2211-2219. 
14. Djozan, D.; Ebrahimi, B.; Mahkam, M.; Farajzadeh, M.A. Evaluation of a new method for chemical coating of aluminum wire with molecularly imprinted polymer layer. Application for the fabrication of triazines selective solid-phase microextraction fiber. Anal. Chim. Acta 2010, 674, 40-48.

15. Aguilera, A.; Rodriguez, M.; Brotons, M.; Boulaid, M.; Valverde, A. Evaluation of supercritical fluid extraction/aminopropyl solid-phase "in-line" cleanup for analysis of pesticide residues in rice. J. Agric. Food Chem. 2005, 53, 9374-9382.

16. Chen, J.; Liu, M.; Zhang, X.; Ding, L.; Yu, Y.; Wang, X.; Jin, H.; Zhang, H. Determination of triazine herbicides in sheep liver by microwave-assisted extraction and high performance liquid chromatography. Anal. Chim. Acta 2007, 590, 34-39.

17. Pang, G.F.; Liu, Y.M.; Fan, C.L.; Zhang, J.J.; Cao, Y.Z.; Li, X.M.; Li, Z.Y.; Wu, Y.P.; Guo, T.T. Simultaneous determination of pesticide residues in grain by accelerated solvent extraction then gas chromatography-mass spectrometry or liquid chromatography-tandem mass spectrometry. Anal. Bioanal. Chem. 2006, 384, 1366-1408.

18. Marchese, S.; Perret, D.; Bafile, E.; Gentili, A.; Caretti, F.; Berardino, M. Pressurized liquid extraction coupled with LC-ESI-MS-MS for the determination of herbicides chlormequat and mepiquat in flours. Chromatographia 2009, 70, 761-769.

19. Sampaio, M.R.F.; Tomasini, D.; Cardoso, L.V.; Caldas, S.S.; Primel, E.G. Determination of pesticide residues in sugarcane honey by QuEChERS and liquid chromatography. J. Braz. Chem. Soc. 2012, 23, 197-205.

20. Jovanov, P.; Guzsvány, V.; Franko, M.; Lazić, S.; Sakač, M.; Šarić, B.; Banjac, V. Multiresidue method for determination of selected neonicotinoid insecticides in honey using optimized dispersive liquid-liquid microextraction combined with liquid chromatographytandem mass spectrometry. Talanta 2013, 111, 125-133.

21. Jovanov, P.; Guzsvány, V.; Lazić, S.; Franko, M. Development of HPLC-DAD method for determination of neonicotinoids in honey. J. Food Compos. Anal. 2015, 40, 106-113.

22. Lorenz, J.G.; Costa, L.L.F.; Suchara, E.A.; Sant'Anna, E.S. Multivariate optimization of the QuEChERS-GC-ECD method and pesticide investigation residues in apples, strawberries, and tomatoes produced in Brazilian south. J. Braz. Chem. Soc. 2014, 25, 1583-1591.

23. He, Z.; Wang, L.; Peng, Y.; Luo, M.; Wang, W.; Liu, X. Multiresidue analysis of over 200 pesticides in cereals using a QuEChERS and gas chromatography-tandem mass spectrometry-based method. Food Chem. 2015, 169, 372-380.

24. Payá, P.; Anastassiades, M.; Mack, D.; Sigalova, I.; Tasdelen, B.; Oliva, J.; Barba, A. Analysis of pesticide residues using the Quick Easy Cheap Effective Rugged and Safe (QuEChERS) pesticide multiresidue method in combination with gas and liquid chromatography and tandem mass spectrometric detection. Anal. Bioanal. Chem. 2007, 389, $1697-1714$

25. Mol, H.J.; Plaza-Bolanos, P.; Zomer, P.; de Rijk, T.C.; Stolker, A.A.M.; Mulder, P.P. Toward a generic extraction method for simultaneous determination of pesticides, mycotoxins, plant toxins, and veterinary drugs in feed and food matrixes. Anal. Chem. 2008, $80,9450-9459$.

26. Takatori, S.; Okihashi, M.; Okamoto, Y.; Kitagawa, Y.; Kakimoto, S.; Murata, H.; Sumimoto, T.; Tanaka, Y.A rapid and easy multiresidue method for the determination of pesticide residues in vegetables, fruits, and cereals using liquid chromatography/tandem mass spectrometry. J. AOAC Int. 2008, 91, 871-883.

27. Nguyen, T.D.; Han, E.M.; Seo, M.S.; Kim, S.R.; Yun, M.Y.; Lee, D.M.; Lee, G.H. A multiresidue method for the determination of 203 pesticides in rice paddies using gas chromatography/mass spectrometry. Anal. Chim. Acta 2008, 619, 67-74. 
28. Mastovska, K.; Dorweiler, K.J.; Lehotay, S.J.; Wegscheid, J.S.; Szpylka, K.A. Pesticide multiresidue analysis in cereal grains using modified QuEChERS method combined with automated direct sample introduction GC-TOFMS and UPLC-MS/MS techniques. J. Agric. Food Chem. 2010, 58, 5959-5972.

29. Kolberg, D.I.; Prestes, O.D.; Adaime, M.B.; Zanella, R. Development of a fast multiresidue method for the determination of pesticides in dry samples (wheat grains, flour and bran) using QuEChERS based method and GC-MS. Food Chem. 2011, 125, 1436-1442.

30. Pareja, L.; Cesio, V.; Heinzen, H.; Fernandez-Alba, A.R. Evaluation of various QuEChERS based methods for the analysis of herbicides and other commonly used pesticides in polished rice by LC-MS/MS. Talanta 2011, 83, 1613-1622.

31. Melo, A.; Mansilha, C.; Pinho, O.; Ferreira, I.M.P.L.V.O. Analysis of pesticides in tomato combining QuEChERS and dispersive liquid-liquid microextraction followed by highperformance liquid chromatography. Food Anal. Methods 2013, 6, 559-568.

32. Bedassa, T.; Gure, A.; Megersa, N. Modified QuEChERS method for the determination of multiclass pesticide residues in fruit samples utilizing high-performance liquid chromatography. Food Anal. Methods 2015, 8, 2020-2027.

33. Walorczyk, S.; Drozdzynski, D. Improvement and extension to new analytes of a multiresidue method for the determination of pesticides in cereals and dry animal feed using gas chromatography-tandem quadrupole mass spectrometry revisited. J. Chromatogr. A 2012, $1251,219-231$

34. Hou, X.; Han, M.; Dai, X..; Yang, X.; Yi, S. A multi-residue method for the determination of 124 pesticides in rice by modified QuEChERS extraction and gas chromatographytandem mass spectrometry. Food Chem. 2013, 138, 1198-1205.

35. Cunha, S.C.; Fernandes, J.O. Multipesticide residue analysis in maize combining acetonitrile-based extraction with dispersive liquid-liquid microextraction followed by gas chromatography-mass spectrometry. J. Chromatogr. A 2011, 1218, 7748-7757.

36. Chen, L.; Yin, L.; Song, F.; Liu, Z.; Zheng, Z.; Xing, J.; Liu, S. Determination of pesticide residues in ginseng by dispersive liquid-liquid microextraction and ultra high performance liquid chromatography-tandem mass spectrometry. J. Chromatogr. B 2013, 917-918, 71-77.

37. Andrascikova, M.; Hrouzkova, S.; Cunha, S.C. Combination of QuEChERS and DLLME for GC-MS determination of pesticide residues in orange samples. Food Addit. Contam. Part A 2013, 30, 286-297.

38. Farajzadeh, M.A.; Feriduni, B.; Mogaddam, M.R.A. Development of a new extraction method based on counter current salting-out homogenous liquid-liquid extraction followed by dispersive liquid-liquid microextraction: Application for the extraction and preconcentration of widely used pesticides from fruit juices. Talanta 2016, 146, 772-779.

39. Zewdu, A.D.; Solomon, S.K. Moisture-dependent physical properties of Tef seed. Biosyst. Eng. 2007, 96, 57-63.

40. Shiferaw, W.; Balcha, A.; Mohammed, H. Evaluation of drought tolerance indices in Tef [Eragrostis tef (Zucc.)Trotter]. Afr. J. Agric. Res. 2012, 7, 3433-3438.

41. Tadesse, B.; Teju, E.; Megersa, N. The Teff straw: a novel low-cost adsorbent for quantitative removal of $\mathrm{Cr}(\mathrm{VI})$ from contaminated aqueous samples. Desalin. Water Treat. 2015, 56, 2925-2936.

42. Rezaee, M.; Yamin, Y.; Faraji, M. Evolution of dispersive liquid-liquid microextraction method. J. Chromatogr. A 2010, 1217, 2342-2357.

43. Zhang, Y.; Lee, H. K. Low-density solvent-based vortex-assisted surfactant-enhancedemulsification liquid-liquid microextraction combined with gas chromatography-mass spectrometry for the fast determination of phthalate esters in bottled water. J. Chromatogr. A 2013, 1274, 28-35. 
44. Ghambari, H.; Hadjmohammadi, M. Low-density solvent-based dispersive liquid-liquid microextraction followed by high performance liquid chromatography for determination of warfarin in human plasma. J. Chromatogr. B 2012, 899, 66-71.

45. Gure, A.; Lara, F.J.; Megersa, N.; del Olmo-Iruela, M.; García-Campañ, A.M. Dispersive liquid-liquid microextraction followed by capillary high-performance liquid chromatography for the determination of six sulfonylurea herbicides in fruit juices. Food Anal. Methods 2014, 7(7), 1465-1473.

46. Tomasini, D.; Sampaio, M.R.F.; Caldas, S.S.; Buffon, J.G.; Duarte, F.A.; Primel, E.G. Simultaneous determination of pesticides and 5-hydroxymethylfurfural in honey by the modified QuEChERS method and liquid chromatography coupled to tandem mass spectrometry. Talanta 2012, 99, 380-386.

47. Wang, P.; Yang, X.; Wang, J.; Cui, J.; Dong, A.J.; Zhao, H.T.; Zhang, L.W.; Wang, Z.Y.; Xu, R.B.; Li, W.J.; Zhang, Y.C.; Zhang, H.; Jing, J. Multi-residue method for determination of seven neonicotinoid insecticides in grains using dispersive solid-phase extraction and dispersive liquid-liquid micro-extraction by high performance liquid chromatography. Food Chem. 2012, 134, 1691-1698.

48. Tolcha, T.; Merdassa, Y.; Megersa, N. Low-density extraction solvent based solventterminated dispersive liquid-liquid microextraction for quantitative determination of ionizable pesticides in environmental waters. J. Sep. Sci. 2013, 36, 1119-1127.

49. Megersa, N. Hollow fiber-liquid phase microextraction for trace enrichment of the residues of atrazine and its major degradation products from environmental water and human urine samples. Anal. Methods 2015, 7, 9940-9948.

50. European Commission Guidance document on analytical quality control and validation procedures for pesticide residues analysis in food and feed. SANCO/12571/2013, Supersedes SANCO/12495/2011, Implemented by 01/01/2014. 